\title{
Payoff Shares in Two-Player Contests
}

\author{
Samuel Häfner and Georg Nöldeke * \\ Faculty of Business and Economics, University of Basel, Peter Merian-Weg 6, Basel 4002, Switzerland; \\ samuel.haefner@unibas.ch \\ * Correspondence: georg.noeldeke@unibas.ch; Tel.: +41-61-207-3320; Fax: +41-61-207-2759 \\ Academic Editor: Ulrich Berger \\ Received: 12 August 2016 ; Accepted: 5 September 2016 ; Published: 9 September 2016
}

\begin{abstract}
In imperfectly discriminating contests with symmetric valuations, equilibrium payoffs are positive shares of the value of the prize. In contrast to a bargaining situation, players' shares sum to less than one because a residual share of the value is lost due to rent dissipation. In this paper, we consider contests with two players and investigate the relationship between these equilibrium shares and the parameters of a class of asymmetric Tullock contest success functions. Our main finding is that any players' shares that sum up to less than one can arise as the unique outcome of a pure-strategy Nash equilibrium for appropriate parameters.
\end{abstract}

Keywords: contests; pure-strategy equilibrium; rent-dissipation

JEL: C72; D72; D74

\section{Introduction}

Contest theory deals with games in which players sink efforts in order to win some prize. A prominent application of the theory is rent-seeking, which goes back at least to the seminal contribution of Tullock [1]. In a rent-seeking contest, the prize consists in a political rent stemming, e.g., from privatization, industry regulation, or protectionist trade policy, and potential beneficiaries of that rent compete by engaging in costly lobbying activities. The literature (extensively surveyed in Konrad [2]) has since identified numerous situations that have a similar contest structure, albeit with different players and prizes, including electoral competition, campaign spending, committee bribing, or militarized disputes.

In this paper, we investigate a contest with a prize of common value $v>0$. The equilibrium of such a contest gives rise to a division of the prize into positive payoff shares to the players that sum up to less than the total prize, and a residual share that is lost due to rent dissipation. Contest theory generally analyzes equilibrium behavior (and thus the resulting division of the prize) for a given specification of the contest success functions-i.e., the map from the efforts of the players into their winning probabilities. Here, we pursue the opposite approach, and ask whether any division of the prize can be supported by the Nash equilibrium of an appropriately-specified contest success function.

To this end, we study pure-strategy Nash equilibria of imperfectly discriminating contests in which risk-neutral players $i$ simultaneously expend efforts $x_{i} \geq 0$ in order to increase their probability $p_{i}$ of winning the single prize. We focus on contests with two players, and consider the class of asymmetric contest success functions having the form $p_{i}=\alpha_{i} x_{i}^{r} /\left(\alpha_{1} x_{1}^{r}+\alpha_{2} x_{2}^{r}\right)$, axiomatized in Clark and Riis [3].

We show that this class of contests is rich enough to support any positive payoffs for the players, summing to less than the value of the prize, as the outcome of a unique pure-strategy Nash equilibrium for appropriately chosen values of the effectiveness parameters $\alpha_{i}>0$ and the decisiveness parameter $r>0$. The proof is constructive in the sense that for every division of the value $v$ into payoff shares and a dissipated share, we provide the parameter values which implement the division. 
One possible interpretation of our result is a negative one: in the absence of any specific information about the structure of the contest, theory does not offer any prediction about equilibrium payoffs going beyond the assertion that these must be feasible and individual rational. Our own interpretation is more positive. We find it natural to think of contests as being embedded in broader economic interactions (e.g., the rent at stake in a rent-seeking contest is not exogenous, but determined by prior investment decisions). It is then convenient to describe a contest in terms of its induced equilibrium payoffs rather than dwelling on the intricacies of the equilibrium analysis of the embedded contest-just as it is often convenient to model bargaining situations in terms of an asymmetric Nash-bargaining solution rather than some non-cooperative game (cf. Chapter 1.2 in Muthoo [4]). But doing so raises the question of whether any specification of equilibrium payoffs can be "micro-founded" through some contest. For the two-player case, our analysis not only resolves this question, but also exhibits the one-to-one relationship between the parameters of the contest success function and the equilibrium payoff shares obtained by the players.

There is an extensive literature discussing the existence and uniqueness of pure-strategy Nash equilibria in n-player contests. Most closely related to our paper are Pérez-Castrillo and Verdier [5], Szidarovszky and Okuguchi [6], and Cornes and Hartley [7]. Pérez-Castrillo and Verdier [5] consider contest success functions of the form $p_{i}=x_{i}^{r} /\left(\sum_{j=1}^{n} x_{j}^{r}\right)$. For $n=2$, this is the special case of our model with a symmetric contest success function $\left(\alpha_{1}=\alpha_{2}\right)$. For this case, it is immediate apparent from the results in Pérez-Castrillo and Verdier [5] that any division in which both players obtain identical, positive equilibrium payoffs summing to less than the prize can be supported by an appropriately chosen decisiveness parameter $r$. Our analysis generalizes this observation by allowing for asymmetric effectiveness parameters. Szidarovszky and Okuguchi [6] establish a sufficient condition for the existence of a unique pure-strategy Nash equilibrium for contest success functions of the ratio-form $p_{i}=f_{i}\left(x_{i}\right) / \sum_{j=1}^{n} f_{j}\left(x_{j}\right)$. For the two-player contests with $f_{i}\left(x_{i}\right)=\alpha_{i} x_{i}^{r}$ we consider, their sufficient condition is satisfied if and only if the decisiveness parameter satisfies $r \leq 1$. We point out a more permissive sufficient condition on $r$ that is also necessary. The condition is crucial for our main result, because it allows us to show that the set of feasible dissipated shares includes all positive shares up to one. Our necessary and sufficient condition for the existence and uniqueness of pure-strategy Nash equilibrium could be inferred using the tools developed in Cornes and Hartley [7], who generalize Szidarovszky and Okuguchi [6] in a number of directions. We offer a simpler direct proof for the two-player case. Further related papers are Baik [8] and Nti [9], who discuss the comparative statics of pure-strategy Nash equilibria in asymmetric contests. Similar to Szidarovszky and Okuguchi [6], these authors impose assumptions on the contest success functions which amount to imposing $r \leq 1$ in our setting, making their results inapplicable for our purposes.

\section{Model}

Risk-neutral players $i=1,2$ simultaneously choose efforts $x_{i} \geq 0$ at cost $x_{i}$. Both players assign value $v>0$ to winning the prize and value 0 to not winning the prize. Player $i^{\prime}$ s payoff function is

$$
U_{i}\left(x_{1}, x_{2}\right)=p_{i}\left(x_{1}, x_{2}\right) \cdot v-x_{i}
$$

where the probability $p_{i}\left(x_{1}, x_{2}\right)$ that player $i$ wins the prize is given by the contest success function

$$
p_{i}\left(x_{1}, x_{2}\right)=\left\{\begin{array}{ll}
\frac{\alpha_{i} x_{i}^{r}}{\alpha_{1} x_{1}^{r}+\alpha_{2} x_{2}^{r}} & \text { if } x_{1}+x_{2}>0 \\
\frac{\alpha_{i}}{\alpha_{1}+\alpha_{2}} & \text { if } x_{1}=x_{2}=0
\end{array},\right.
$$

with $\alpha_{i}>0$ and $r>0$. (Our main result, Proposition 1, remains unchanged for any specification of $p_{i}(0,0) \geq 0$ satisfying $p_{1}(0,0)+p_{2}(0,0) \leq 1$.) As the payoff functions are homogeneous of degree 
zero in $\alpha_{1}$ and $\alpha_{2}$, it is without loss of generality to assume $\alpha_{1}+\alpha_{2}=1$, and we will do so throughout the following. Let

$$
\mathcal{P}=\left\{\left(\alpha_{1}, \alpha_{2}, r\right) \in \mathbb{R}_{++}^{3}: \alpha_{1}+\alpha_{2}=1\right\}
$$

denote the corresponding set of feasible parameters for the contest success function. The parameters of the contest are then given by the quadruple $\left(\alpha_{1}, \alpha_{2}, r, v\right) \in \mathcal{P} \times \mathbb{R}_{++}$. A pure-strategy Nash equilibrium (or simply equilibrium) of such a contest is a strategy profile $\left(x_{1}^{*}, x_{2}^{*}\right) \in \mathbb{R}_{+}^{2}$ satisfying

$$
x_{1}^{*} \in \underset{x_{1} \geq 0}{\operatorname{argmax}} U_{1}\left(x_{1}, x_{2}^{*}\right) \text { and } x_{2}^{*} \in \underset{x_{2} \geq 0}{\operatorname{argmax}} U_{2}\left(x_{1}^{*}, x_{2}\right) .
$$

Every equilibrium $\left(x_{1}^{*}, x_{2}^{*}\right)$ gives rise to a division of the value of the prize into equilibrium payoffs $u_{i}^{*}=U_{i}\left(x_{1}^{*}, x_{2}^{*}\right)$ for the two players, and a rent-dissipation term $d^{*}=v-u_{1}^{*}-u_{2}^{*}$. Each player $i$ can assure a positive payoff by choosing the strategy $x_{i}=0$, and for any strategy combination $\left(x_{1}, x_{2}\right)$, the sum of the two players' payoffs is less than $v$. Hence, for every equilibrium $\left(x_{1}^{*}, x_{2}^{*}\right)$, there exist $\left(s_{1}^{*}, s_{2}^{*}, s_{3}^{*}\right) \in \Delta$, where

$$
\Delta=\left\{\left(s_{1}, s_{2}, s_{3}\right) \in \mathbb{R}_{+}^{3}: s_{1}+s_{2}+s_{3}=1\right\},
$$

such that $u_{1}^{*}=s_{1}^{*} \cdot v, u_{2}^{*}=s_{2}^{*} \cdot v$, and $d^{*}=s_{3}^{*} \cdot v$. That is, we can view any equilibrium $\left(x_{1}^{*}, x_{2}^{*}\right)$ of the contest as inducing a division of the value of the prize into payoff shares $s_{1}^{*}$ and $s_{2}^{*}$ for the two contestants, and a dissipated share $s_{3}^{*}$. We refer to these shares as equilibrium shares. We find it convenient to phrase our analysis and results in terms of equilibrium shares rather than equilibrium payoffs.

\section{Results}

We begin by stating our main result:

Proposition 1. For any $\left(s_{1}^{*}, s_{2}^{*}, s_{3}^{*}\right) \in \Delta$ satisfying $s_{3}^{*}>0$, there exists a unique $\left(\alpha_{1}, \alpha_{2}, r\right) \in \mathcal{P}$ such that any contest with parameters $\left(\alpha_{1}, \alpha_{2}, r, v\right)$ has a unique pure-strategy Nash equilibrium $\left(x_{1}^{*}, x_{2}^{*}\right)$ with equilibrium shares $\left(s_{1}^{*}, s_{2}^{*}, s_{3}^{*}\right)$.

The following two lemmas establish Proposition 1. Lemma 1 delineates the set $\mathcal{P}^{*} \subset \mathcal{P}$ of parameters of the contest success function for which a unique equilibrium exists and determines the equilibrium strategies and shares as functions of the parameters. Lemma 2 then completes the proof by exhibiting — for any shares in $\Delta$ satisfying $s_{3}>0$ — the unique parameters in $\mathcal{P}^{*}$ yielding these shares as equilibrium shares.

Lemma 1. A contest with parameters $\left(\alpha_{1}, \alpha_{2}, r, v\right) \in \mathcal{P} \times \mathbb{R}_{++}$has a pure-strategy Nash equilibrium if and only if $\left(\alpha_{1}, \alpha_{2}, r\right) \in \mathcal{P}^{*}$ holds, where

$$
\mathcal{P}^{*}=\left\{\left(\alpha_{1}, \alpha_{2}, r\right) \in \mathcal{P}: r \leq \frac{1}{\max \left\{\alpha_{1}, \alpha_{2}\right\}}\right\} .
$$

If a pure-strategy Nash equilibrium exists, it is unique with equilibrium efforts

$$
x_{1}^{*}=x_{2}^{*}=\alpha_{1} \alpha_{2} r v
$$

and equilibrium shares

$$
\begin{aligned}
& s_{1}^{*}=\alpha_{1}-\alpha_{1} \alpha_{2} r \\
& s_{2}^{*}=\alpha_{2}-\alpha_{1} \alpha_{2} r \\
& s_{3}^{*}=2 \alpha_{1} \alpha_{2} r .
\end{aligned}
$$


Most of the proof of Lemma 1 (which we have relegated to the appendix) is straightforward. Taking the existence of equilibrium for granted, uniqueness can be established directly by considering the appropriate first order conditions. As shown by Mills [10], these imply that the equilibrium efforts are identical and given by (7). Equations (8) and (9) for the equilibrium payoff shares $s_{1}^{*}$ and $s_{2}^{*}$ are then immediate: with equal efforts, the probability that contestant $i$ wins the prize is $\alpha_{i}$, so that $s_{i}^{*}=\alpha_{i}-x_{i}^{*} / v$. Similarly, equal equilibrium efforts imply that the dissipated rent is twice the individual effort, yielding the expression for $s_{3}^{*}$ in (10). Further, as equilibrium payoff shares must be positive, it is apparent from (8) and (9) that $r \leq 1 / \max \left\{\alpha_{1}, \alpha_{2}\right\}$ is necessary for the existence of equilibrium. Showing that the same condition suffices for the existence of equilibrium is more subtle, because for $r>1$, players' payoff functions are not concave in their own efforts. Therefore, some care is required in showing that the effort levels $\left(x_{1}^{*}, x_{2}^{*}\right)$ solving the first order conditions are indeed an equilibrium.

It is not difficult to see that for any shares $\left(s_{1}^{*}, s_{2}^{*}, s_{3}^{*}\right) \in \Delta$ satisfying $s_{3}^{*}>0$, the system of Equations (8)-(10) can be inverted to solve for uniquely determined parameter values $\left(\alpha_{1}, \alpha_{2}, r\right) \in \mathcal{P}$ of the underlying contest success function. To establish Proposition 1 we also have to show that for these parameter values, a pure-strategy Nash equilibrium does indeed exist. That is, we have to show that $\left(\alpha_{1}, \alpha_{2}, r\right) \in \mathcal{P}^{*}$ holds. This is accomplished in the proof of the following lemma, which we have again relegated to the appendix.

Lemma 2. For any $\left(s_{1}^{*}, s_{2}^{*}, s_{3}^{*}\right) \in \Delta$ satisfying $s_{3}^{*}>0$, there exists a unique $\left(\alpha_{1}, \alpha_{2}, r\right) \in \mathcal{P}^{*}$, given by

$$
\begin{aligned}
\alpha_{1} & =\frac{1+s_{1}^{*}-s_{2}^{*}}{2} \\
\alpha_{2} & =\frac{1+s_{2}^{*}-s_{1}^{*}}{2} \\
r & =\frac{2 s_{3}^{*}}{\left(1+s_{1}^{*}-s_{2}^{*}\right)\left(1+s_{2}^{*}-s_{1}^{*}\right)},
\end{aligned}
$$

such that Equations (8)-(10) hold.

The result that the parameters of the contest success function can be uniquely determined from the equilibrium shares is, of course, due to our assumption that the effectiveness parameters are normalized to satisfy $\alpha_{1}+\alpha_{2}=1$. Without this normalization, the right side of Equation (11) equals $\alpha_{1} /\left(\alpha_{1}+\alpha_{2}\right)$ and, similarly, the right side of Equation (12) can be used to infer the share of Player 2's effectiveness parameter. On the other hand, our normalization plays no role in the determination of the decisiveness parameter $r$, which can always be uniquely inferred from the equilibrium shares via (13). Similarly, the equilibrium efforts can always be inferred from knowledge of the dissipated share via the relationship $x_{1}^{*}=x_{2}^{*}=s_{3}^{*} /(2 v)$, which is a direct consequence of the equality of the equilibrium efforts and thus does not hinge on our normalization.

\section{Discussion and Conclusions}

For a given value of the prize $v>0$, our analysis establishes that any payoffs satisfying $u_{1}^{*} \geq 0$, $u_{2}^{*} \geq 0$, and $u_{1}^{*}+u_{2}^{*}<v$ can arise as the unique equilibrium payoffs in a two-player contest with contest success function $p_{i}=\alpha_{i} x_{i}^{r} /\left(\alpha_{1} x_{1}^{r}+\alpha_{2} x_{2}^{r}\right)$. Even though we have excluded the trivial cases $r=0, \alpha_{1}=0$, and $\alpha_{2}=0$ from our formal analysis, it is clear that allowing for these possibilities implies that all feasible and individually rational payoffs can be obtained as equilibrium payoffs. It is then an immediate implication that allowing for more general contest success function (e.g., of the ratio-form $p_{i}=f_{i}\left(x_{i}\right) /\left(f_{1}\left(x_{1}\right)+f_{2}\left(x_{2}\right)\right)$ considered in [6] and [7]) does not enlarge the set of possible equilibrium payoffs beyond what can be obtained with the simple contest success functions we consider. This observation is reminiscent of Proposition 1 in Dasgupta and Nti [11]. These authors 
consider a class of symmetric contest success functions of the form $p_{i}=h\left(x_{i}\right) /\left(s+\sum_{j=0}^{n} h\left(x_{j}\right)\right)$, where the introduction of the parameter $s \geq 0$ allows for the possibility that none of the contestants obtains the prize and the function $h$ is assumed increasing and concave. Proposition 1 of their paper shows that any equilibrium outcome arising from some contest success function in this class can also be obtained as an equilibrium outcome of a contest in which the function $h$ is linear.

The special form of the contest success function we consider ensures that-as indicated by Lemma 1 -for given parameters $\left(\alpha_{1}, \alpha_{2}, r\right) \in \mathcal{P}^{*}$ of the contest success function, the resulting equilibrium shares $\left(s_{1}^{*}, s_{2}^{*}, s_{3}^{*}\right)$ are independent of the value of the prize $v$. As a consequence of this property, Proposition 1 may be interpreted as establishing that any linear sharing rule (in which players receive a positive share of the prize as a payoff and a strictly positive share of the prize is dissipated) can arise as an equilibrium outcome of the asymmetric two-person contest we consider. Given the simplicity of incorporating linear sharing rules as a building block in more elaborate economic models, we view this result as an attractive feature of our model. Linearity of the sharing rule holds because, as required by Axiom A6 both in Skaperdas [12] and Clark and Riis [3], the contest success functions we consider are homogeneous of degree zero in effort. Considering more general contest success functions, like the ones mentioned in the previous paragraph, will destroy this feature.

Acknowledgments: Financial support by the Swiss National Science Foundation (Grant 146089) is gratefully acknowledged.

Author Contributions: The authors contributed equally to the design of the model, its analysis, and the writing of the paper.

Conflicts of Interest: The authors declare no conflict of interest.

\section{Appendix}

Proof of Lemma 1. There can be no equilibrium with either or both efforts equal to zero: Suppose, without loss of generality, that $x_{2}=0$. Then, we have $U_{1}\left(x_{1}, x_{2}\right)=1-x_{1}$ for $x_{1}>0$ and $U_{1}\left(0, x_{2}\right)=\alpha_{1}<1$, so that Player 1 has no best response. Hence, every equilibrium satisfies $\left(x_{1}, x_{2}\right) \in \mathbb{R}_{++}^{2}$. As the payoff functions are differentiable on $\mathbb{R}_{++}^{2}$, the first order conditions

$$
\begin{aligned}
& \frac{\partial U_{1}\left(x_{1}, x_{2}\right)}{\partial x_{1}}=\frac{\alpha_{1} \alpha_{2} r x_{1}^{r-1} x_{2}^{r}}{\left(\alpha_{1} x_{1}^{r}+\alpha_{2} x_{2}^{r}\right)^{2}} v-1=0 \\
& \frac{\partial U_{2}\left(x_{1}, x_{2}\right)}{\partial x_{2}}=\frac{\alpha_{1} \alpha_{2} r x_{2}^{r-1} x_{1}^{r}}{\left(\alpha_{1} x_{1}^{r}+\alpha_{2} x_{2}^{r}\right)^{2}} v-1=0
\end{aligned}
$$

are then necessary for $\left(x_{1}, x_{2}\right)$ to be an equilibrium. This yields $x_{1}=x_{2}$. Substituting back into (14) and (15), we obtain $\left(x_{1}^{*}, x_{2}^{*}\right)$ as given in (7) as the unique candidate for an equilibrium with corresponding equilibrium utilities

$$
\begin{aligned}
& u_{1}^{*}=U_{1}\left(x_{1}^{*}, x_{2}^{*}\right)=\left[\alpha_{1}-\alpha_{1} \alpha_{2} r\right] v \\
& u_{2}^{*}=U_{2}\left(x_{1}^{*}, x_{2}^{*}\right)=\left[\alpha_{2}-\alpha_{1} \alpha_{2} r\right] v .
\end{aligned}
$$

The expressions for the equilibrium shares in (8)-(10) are then immediate.

Because player $i$ can secure a payoff of zero by choosing $x_{i}=0$, any equilibrium must satisfy $\min \left\{u_{1}^{*}, u_{2}^{*}\right\} \geq 0$. From (16) and (17), this condition is equivalent to $r \leq 1 / \max \left\{\alpha_{1}, \alpha_{2}\right\}$. To finish 
the proof, it remains to show that this condition is also sufficient for $\left(x_{1}^{*}, x_{2}^{*}\right)$ to be an equilibrium. Towards this end, consider the second derivatives (well-defined on $\mathbb{R}_{++}^{2}$ )

$$
\begin{aligned}
& \frac{\partial^{2} U_{1}\left(x_{1}, x_{2}\right)}{\partial x_{1}^{2}}=A_{1}\left(x_{1}, x_{2}\right)\left[\alpha_{2}(r-1) x_{2}^{r}-\alpha_{1}(r+1) x_{1}^{r}\right] \\
& \frac{\partial^{2} U_{2}\left(x_{1}, x_{2}\right)}{\partial x_{2}^{2}}=A_{2}\left(x_{1}, x_{2}\right)\left[\alpha_{1}(r-1) x_{1}^{r}-\alpha_{2}(r+1) x_{2}^{r}\right],
\end{aligned}
$$

where $A_{1}\left(x_{1}, x_{2}\right)=\left(\alpha_{1} \alpha_{2} r x_{1}^{r-2} x_{2}^{r} v\right) /\left(\alpha_{1} x_{1}^{r}+\alpha_{2} x_{2}^{r}\right)^{3}>0$ and $A_{2}\left(x_{1}, x_{2}\right)=\left(\alpha_{1} \alpha_{2} r x_{2}^{r-2} x_{1}^{r} v\right) /\left(\alpha_{1} x_{1}^{r}+\alpha_{2} x_{2}^{r}\right)^{3}>0$. The signs of these derivatives are equal to the signs of the terms in square brackets in (18) and (19). For $0<r \leq 1$, these terms are strictly negative, so that players' payoff functions are concave in their own efforts, ensuring that the solution to the first order conditions (14) and (15) satisfies the equilibrium conditions (4). For $r>1$, the terms in square brackets in (18) and (19) have exactly one sign change in $x_{1}$ and $x_{2}$, respectively, from positive to negative, so that the same holds for the second derivatives. Consequently, the first derivatives of players' payoff functions are unimodal (first increasing, then decreasing) in their own efforts. Hence, if (for given $x_{2}>0$ ) the first order condition (14) has a solution $\hat{x}_{1}>0$ satisfying $U_{1}\left(\hat{x}_{1}, x_{2}\right) \geq U_{1}\left(0, x_{2}\right)=0$, then this solution solves $\max _{x_{1} \geq 0} U_{1}\left(x_{1}, x_{2}\right)$ and, similarly, if (for given $x_{1}>0$ ) the first order condition (15) has a solution $\hat{x}_{2}>0$ satisfying $U_{2}\left(x_{1}, \hat{x}_{2}\right) \geq U_{1}\left(x_{1}, 0\right)=0$, then this solution solves $\max _{x_{2} \geq 0} U_{2}\left(x_{1}, x_{2}\right)$. From this, the desired result is immediate.

Proof of Lemma 2. First, we show that $\left(\alpha_{1}, \alpha_{2}, r\right)$ as given by (11)-(13) is in $\mathcal{P}^{*}$ : Adding Equations (11) and (12) yields $\alpha_{1}+\alpha_{2}=1$. From $\left(s_{1}^{*}, s_{2}^{*}, s_{3}^{*}\right) \in \Delta$ and $s_{3}^{*}>0$, we have $\left|s_{2}^{*}-s_{1}^{*}\right|<1$, so that (11)-(13) imply $\alpha_{1}>0, \alpha_{2}>0$, and $r>0$. Hence, $\left(\alpha_{1}, \alpha_{2}, r\right) \in \mathcal{P}$. Given (11) and (12), Equation (13) can be written as

$$
r=\frac{s_{3}^{*}}{2 \alpha_{1} \alpha_{2}}=\frac{s_{3}^{*}}{2 \min \left\{\alpha_{1}, \alpha_{2}\right\} \max \left\{\alpha_{1}, \alpha_{2}\right\}}
$$

Because

$$
s_{3}^{*}=1-s_{1}^{*}-s_{2}^{*} \leq \min \left\{1+s_{1}^{*}-s_{2}^{*}, 1+s_{2}^{*}-s_{1}^{*}\right\}=2 \min \left\{\alpha_{1}, \alpha_{2}\right\}
$$

Equation (20) then implies $r \leq 1 / \max \left\{\alpha_{1}, \alpha_{2}\right\}$, yielding $\left(\alpha_{1}, \alpha_{2}, r\right) \in \mathcal{P}^{*}$.

Second, we show that the parameter values given in (11)-(13) are the unique parameter values in $\mathcal{P}^{*}$ such that (8)-(10) hold. Replacing $\left(\alpha_{1}, \alpha_{2}, r\right)$ in (8)-(10) by the expressions on the right sides of (11)-(13) and simplifying shows that Equations (8)-(10) are satisfied. Vice versa, suppose that for $\left(\alpha_{1}, \alpha_{2}, r\right) \in \mathcal{P}^{*}$, Equations (8)-(10) hold. Subtracting Equation (9) from Equation (8), we find $\alpha_{1}-\alpha_{2}=s_{1}^{*}-s_{2}^{*}$. Using $\alpha_{1}+\alpha_{2}=1$, this yields (11) and (12). From (10), we have $r=s_{3}^{*} /\left(2 \alpha_{1} \alpha_{2}\right)$. Replacing $\alpha_{1}$ and $\alpha_{2}$ by the right sides of (11) and (12) then yields (13).

\section{References}

1. Tullock, G. Efficient rent seeking. In Toward a Theory of the Rent-Seeking Society; Buchanan, J.M., Tollison, R.D., Tullock, G., Eds.; Texas A \& M University Press: College Station, TX, USA, 1980.

2. Konrad, K.A. Strategy and Dynamics in Contests; Oxford University Press: Oxford, UK, 2009.

3. Clark, D.J.; Riis, C. Contest Success Functions: An Extension. Econ. Theory 1998, 11, 201-204.

4. Muthoo, A. Bargaining Theory with Applications; Cambridge University Press: Cambridge, UK, 1999.

5. Pérez-Castrillo, J.D.; Verdier, T. A General Analysis of Rent-Seeking Games. Public Choice 1992, 73, 335-350.

6. Szidarovszky, F.; Okuguchi, K. On the Existence and Uniqueness of Pure Nash Equilibrium in Rent-Seeking Games. Games Econ. Behav. 1997, 18, 135-140.

7. Cornes, R.; Hartley, R. Asymmetric Contests with General Technologies. Econ. Theory 2005, 26, 923-946.

8. Baik, K.H. Effort Levels in Contests with Two Asymmetric Players. South. Econ. J. 1994, 61, 367-378.

9. Nti, K.O. Comparative Statics of Contests and Rent-Seeking Games. Int. Econ. Rev. 1997, 38, 43-59. 
10. Mills, H.D. A Study in Promotional Competition; Research Paper 101-03; Mathematica: Princeton, NJ, USA, 1959.

11. Dasgupta, A.; Nti, K.O. Designing an Optimal Contest. Eur. J. Political Econ. 1998, 14, 587-603.

12. Skaperdas, S. Contest Success Functions. Econ. Theory 1996, 7, 283-290.

(C) 2016 by the authors; licensee MDPI, Basel, Switzerland. This article is an open access article distributed under the terms and conditions of the Creative Commons Attribution (CC-BY) license (http:/ / creativecommons.org/licenses/by/4.0/). 